Absolute rate coefficients for photorecombination of beryllium-like and boron-like silicon ions

This content has been downloaded from IOPscience. Please scroll down to see the full text.

2016 J. Phys. B: At. Mol. Opt. Phys. 49074004

(http://iopscience.iop.org/0953-4075/49/7/074004)

View the table of contents for this issue, or go to the journal homepage for more

Download details:

IP Address: 128.59.168.116

This content was downloaded on 22/03/2016 at 13:19

Please note that terms and conditions apply. 


\title{
Absolute rate coefficients for photorecombination of beryllium-like and boron-like silicon ions
}

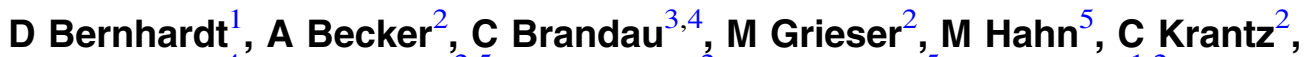 \\ M Lestinsky ${ }^{4}$, O Novotný ${ }^{2,5}$, R Repnow ${ }^{2}$, D W Savin ${ }^{5}$, K Spruck ${ }^{1,2}$, A Wolf ${ }^{2}$, \\ A Müller ${ }^{1}$ and S Schippers ${ }^{3}$ \\ ${ }^{1}$ Institut für Atom- und Molekülphysik, Justus-Liebig-Universität Gießen, Leihgesterner Weg 217, \\ 35392 Giessen, Germany \\ ${ }^{2}$ Max-Planck-Institut für Kernphysik, Saupfercheckweg 1, 69117 Heidelberg, Germany \\ ${ }^{3}$ I. Physikalisches Institut, Justus-Liebig-Universität Gießen, Heinrich-Buff-Ring 16, 35392 Giessen, \\ Germany \\ ${ }^{4}$ GSI Helmholtzzentrum für Schwerionenforschung, Planckstraße 1, 64291 Darmstadt, Germany \\ ${ }^{5}$ Columbia Astrophysics Laboratory, Columbia University, New York, NY 10027, USA \\ E-mail: Stefan.Schippers@physik.uni-giessen.de
}

Received 9 November 2015, revised 22 January 2016

Accepted for publication 5 February 2016

Published 17 March 2016

\begin{abstract}
We report measured rate coefficients for electron-ion recombination of $\mathrm{Si}^{10+}$ forming $\mathrm{Si}^{9+}$ and of $\mathrm{Si}^{9+}$ forming $\mathrm{Si}^{8+}$, respectively. The measurements were performed using the electron-ion merged-beams technique at a heavy-ion storage ring. Electron-ion collision energies ranged from 0 to $50 \mathrm{eV}$ for $\mathrm{Si}^{9+}$ and from 0 to $2000 \mathrm{eV}$ for $\mathrm{Si}^{10+}$, thus, extending previous measurements for $\mathrm{Si}^{10+}$ (Orban et al 2010 Astrophys. J. 721 1603) to much higher energies. Experimentally derived rate coefficients for the recombination of $\mathrm{Si}^{9+}$ and $\mathrm{Si}^{10+}$ ions in a plasma are presented along with simple parameterizations. These rate coefficients are useful for the modeling of the charge balance of silicon in photoionized plasmas $\left(\mathrm{Si}^{9+}\right.$ and $\left.\mathrm{Si}^{10+}\right)$ and in collisionally ionized plasmas ( $\mathrm{Si}^{10+}$ only). In the corresponding temperature ranges, the experimentally derived rate coefficients agree with the latest corresponding theoretical results within the experimental uncertainties.
\end{abstract}

Keywords: atomic processes in plasmas, silicon, electron-ion recombination, heavy-ion storage ring, dielectronic recombination

(Some figures may appear in colour only in the online journal)

\section{Introduction}

In the Universe, silicon is produced by stellar nucleosynthesis and supernova explosions. It is the eighth most abundant element in the solar system [1] and also contributes significantly to the elemental composition of the intergalactic medium [2]. Consequently, prominent photoemission lines from silicon ions are observed from many cosmic plasmas such as the solar corona [3], stellar atmospheres [4], supernova remnants [5], active galactic nuclei [6], and quasars at high redshift [7].
Astrophysical model calculations are required in order to infer the physical conditions of cosmic objects from their observed spectra. The photon emission from any plasma strongly depends on the ionization balances of all abundant atomic species [8, 9]. These, in turn, are governed by ionization and recombination processes. The needed cross sections and rate coefficients are obtained from theoretical calculations which bear (usually unknown) uncertainties [10]. Thus, benchmarking by experiments is required to test the theoretical calculations and to guide the further development of the theoretical methods. 
Table 1. Excitation energies and lifetimes of $\mathrm{Si}^{10+}$ levels. Numbers in brackets denote powers of 10 .

\begin{tabular}{lccc}
\hline & \multicolumn{2}{c}{ Excitation energy } & Lifetime \\
\cline { 2 - 4 } Level & NIST [15] & \multicolumn{2}{c}{ Wang et al $[16]$} \\
\cline { 2 - 4 } & $(\mathrm{eV})$ & $(\mathrm{eV})$ & $(\mathrm{s})$ \\
\hline $1 s^{2} 2 s^{2}{ }^{1} S_{0}$ & 0.00000 & 0.00000 & $\infty$ \\
$1 s^{2} 2 s 2 p{ }^{3} P_{0}$ & 21.0528 & 21.0558 & $1.664[+01]^{\mathrm{a}}$ \\
$1 s^{2} 2 s 2 p{ }^{3} P_{1}$ & 21.3431 & 21.3451 & $2.747[-06]$ \\
$1 s^{2} 2 s 2 p{ }^{3} P_{2}$ & 21.9846 & 21.9876 & $4.729[-01]$ \\
$1 s^{2} 2 s 2 p{ }^{1} P_{1}$ & 40.8750 & 40.8605 & $1.567[-10]$ \\
$1 s^{2} 2 p^{2}{ }^{3} P_{0}$ & 55.0081 & 55.0008 & $2.034[-10]$ \\
$1 s^{2} 2 p^{2}{ }^{3} P_{1}$ & 55.3582 & 55.3526 & $2.001[-10]$ \\
$1 s^{2} 2 p^{2}{ }^{3} P_{2}$ & 55.9125 & 55.9056 & $1.966[-10]$ \\
$1 s^{2} 2 p^{2}{ }^{1} D_{2}$ & 61.3971 & 61.3620 & $9.107[-10]$ \\
$1 s^{2} 2 p^{2}{ }^{1} S_{0}$ & 75.4764 & 75.4172 & $1.042[-10]$ \\
$1 s^{2} 2 s 3 s{ }^{3} S_{1}$ & 274.092 & 274.008 & $3.777[-12]$ \\
$1 s^{2} 2 s 3 s{ }^{1} S_{0}$ & 277.949 & 277.884 & $1.194[-11]$ \\
$1 s^{2} 2 s 3 p{ }^{1} P_{1}$ & 283.309 & 283.351 & $1.495[-12]$ \\
$1 s^{2} 2 s 3 p{ }^{3} P_{0}$ & 283.330 & 283.747 & $2.894[-10]$ \\
$1 s^{2} 2 s 3 p{ }^{3} P_{1}$ & 283.330 & 283.857 & $2.531[-11]$ \\
$1 s^{2} 2 s 3 p{ }^{3} P_{2}$ & 283.330 & 284.005 & $2.658[-10]$ \\
$1 s^{2} 2 s 3 d{ }^{3} D_{1}$ & & 289.109 & $7.488[-13]$ \\
$1 s^{2} 2 s 3 d{ }^{3} D_{2}$ & 289.137 & 289.138 & $7.515[-13]$ \\
$1 s^{2} 2 s 3 d{ }^{3} D_{3}$ & 289.196 & 289.192 & $7.554[-13]$ \\
$1 s^{2} 2 s 3 d{ }^{1} D_{2}$ & 292.763 & 292.731 & $1.137[-12]$ \\
$1 s 2 s^{2} 2 p{ }^{1} P_{1}$ & 1828.618 & & \\
\hline${ }^{2}$ & & & \\
\hline
\end{tabular}

${ }^{a}$ lifetime associated with the hyperfine induced transition taken from [17].

Within our general effort to provide reliable rate coefficients for the photorecombination (PR) and electron-impact ionization (EII) of astrophysically relevant atomic ions [1114] we here publish experimental rate coefficients for the $P R$ of $\mathrm{Si}^{10+}$ forming $\mathrm{Si}^{9+}$ and of $\mathrm{Si}^{9+}$ forming $\mathrm{Si}^{8+}$. For the measurements the electron-ion merged-beams technique was employed at a heavy-ion storage ring.

For Be-like $\mathrm{Si}^{10+}$, the experimental electron-ion collision energy range was $0-2000 \mathrm{eV}$. The most significant photorecombination channels in this energy range are

$$
\begin{aligned}
& \mathrm{Si}^{10+}\left(2 s^{2}\right)+e^{-} \rightarrow \\
& \left\{\begin{array}{llll}
\mathrm{Si}^{9+}\left(2 s^{2} n l\right)+\gamma & & \mathrm{RR} \\
\mathrm{Si}^{9+}(2 s 2 p n l) & \rightarrow \mathrm{Si}^{9+}+\gamma & \Delta N=0 & \mathrm{DR}(2 s \rightarrow 2 p) \\
\mathrm{Si}^{9+}\left(2 p^{2} n l\right) & \rightarrow \mathrm{Si}^{9+}+\gamma & \Delta N=0 & \mathrm{TR}\left(2 s^{2} \rightarrow 2 p^{2}\right) \\
\mathrm{Si}^{9+}\left(2 s 3 l^{\prime} n l\right) & \rightarrow \mathrm{Si}^{9+}+\gamma & \Delta N=1 & \mathrm{DR}\left(2 s \rightarrow 3 l^{\prime}\right) \\
\mathrm{Si}^{9+}\left(2 s 4 l^{\prime} n l\right) & \rightarrow \mathrm{Si}^{9+}+\gamma \quad \Delta N=2 & \mathrm{DR}\left(2 s \rightarrow 4 l^{\prime}\right) \\
\mathrm{Si}^{9+}\left(1 s 2 s^{2} N^{\prime} l^{\prime} n l\right) & \rightarrow \mathrm{Si}^{9+}+\gamma \quad \Delta N>0 & \mathrm{DR}\left(1 s \rightarrow N^{\prime} l^{\prime}\right)
\end{array}\right.
\end{aligned}
$$

where $\gamma$ stands for one or more photons, and RR, DR, and TR denote radiative, dielectronic, and trielectronic recombination, respectively. The intermediate DR and TR resonance states are populated by resonant electron capture of the initially free electron into a subshell $n l$. This capture is associated with the excitation of one (in case of DR) or two (in case of TR) core electrons to a higher core level. Some corresponding core excitation energies are listed in table 1 . The active core electron may be excited such that its principal quantum
Table 2. Excitation energies and lifetimes of $\mathrm{Si}^{9+}$ levels. Numbers in

\begin{tabular}{|c|c|c|c|}
\hline \multirow{3}{*}{ Level } & \multicolumn{2}{|c|}{ Excitation energy } & Lifetime \\
\hline & \multirow{2}{*}{$\begin{array}{c}\text { NIST [15] } \\
(\mathrm{eV})\end{array}$} & \multicolumn{2}{|c|}{ 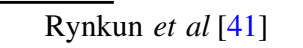 } \\
\hline & & $(\mathrm{eV})$ & (s) \\
\hline $1 s^{2} 2 s^{2} 2 p{ }^{2} P_{1 / 2}$ & 0.0000 & 0.0000 & $\infty$ \\
\hline $1 s^{2} 2 s^{2} 2 p{ }^{2} P_{3 / 2}$ & 0.8667 & 0.8679 & $3.245[-01]$ \\
\hline $1 s^{2} 2 s 2 p^{2}{ }^{4} P_{1 / 2}$ & 19.9627 & 19.9556 & $1.870[-06]$ \\
\hline $1 s^{2} 2 s 2 p^{2}{ }^{4} P_{3 / 2}$ & 20.2702 & 20.2702 & $1.389[-05]$ \\
\hline $1 s^{2} 2 s 2 p^{2}{ }^{4} P_{5 / 2}$ & 20.7128 & 20.7128 & $4.066[-06]$ \\
\hline $1 s^{2} 2 s 2 p^{2}{ }^{2} D_{3 / 2}$ & 35.6888 & 35.7071 & $4.550[-10]$ \\
\hline $1 s^{2} 2 s 2 p^{2}{ }^{2} D_{5 / 2}$ & 35.6926 & 35.6926 & $4.909[-10]$ \\
\hline $1 s^{2} 2 s 2 p^{2}{ }^{2} S_{1 / 2}$ & 45.5853 & 45.6191 & $1.087[-10]$ \\
\hline $1 s^{2} 2 s 2 p^{2}{ }^{2} P_{1 / 2}$ & 48.3588 & 48.3897 & $5.947[-11]$ \\
\hline $1 s^{2} 2 s 2 p^{2}{ }^{2} P_{3 / 2}$ & 48.8535 & 48.8535 & $5.816[-11]$ \\
\hline
\end{tabular}
brackets denote powers of 10 .

number $N$ does not change $(\Delta N=0)$ or such that $N$ changes by $1(\Delta N=1)$ or more $(\Delta N>1)$. DR (and TR) is completed when the multiply excited intermediate level has decayed by photon emission to a bound level of the recombined ion below the first autoionization threshold.

For B-like $\mathrm{Si}^{9+}$ the collision energy range was limited to $0-51 \mathrm{eV}$ because of time constraints. The most important photorecombination channels in this energy range are

$\mathrm{Si}^{9+}\left(2 s^{2} 2 p\right)+e^{-} \rightarrow$

$\left\{\begin{array}{lll}\mathrm{Si}^{8+}\left(2 s^{2} 2 p n l\right)+\gamma & & \mathrm{RR} \\ \mathrm{Si}^{8+}\left(2 s^{2} 2 p n l\right) & \rightarrow \mathrm{Si}^{8+}+\gamma \quad \Delta N=0 & \mathrm{DR}(2 p \rightarrow 2 p) \\ \mathrm{Si}^{8+}\left(2 s 2 p^{2} n l\right) & \rightarrow \mathrm{Si}^{8+}+\gamma \quad \Delta N=0 & \mathrm{DR}(2 s \rightarrow 2 p) .\end{array}\right.$

The excitation energies of the core excitations associated with $\Delta N=0 \mathrm{DR}$ are listed in table 2.

Results from storage-ring experiments have been reported for many ions from the beryllium isoelectronic sequence, i.e, for $\mathrm{C}^{2+}[18], \mathrm{N}^{3+}$ [18], $\mathrm{O}^{4+}$ [18], $\mathrm{F}^{5+}$ [19], $\mathrm{Ne}^{6+}$ [20], $\mathrm{Mg}^{8+}$ [21], Si ${ }^{10+}$ [22], S${ }^{12+}$ [23], $\mathrm{Cl}^{13+}$ [24], $\mathrm{Ti}^{18+}[25,26]$, $\mathrm{Fe}^{22+}$ [27], $\mathrm{Ge}^{28+}$ [28], and $\mathrm{Xe}^{50+}$ [29]. It is noted that the significance of TR was first discovered for Be-like $\mathrm{Cl}^{13+}$ [24] and subsequently confirmed for other ions from this isoelectronic sequence [19-22]. The boron isoelectronic sequence has been less intensely studied. DR rate coefficients from storage-ring experiments are available for $\mathrm{C}^{+}$[30], $\mathrm{Ne}^{3+}$ [31], $\mathrm{Mg}^{7+}[32], \mathrm{Ar}^{13+}[33,34]$, and $\mathrm{Fe}^{21+}[35,36]$. State-of-theart theoretical DR (and TR) calculations have been carried out by Colgan et al [37] and by Altun et al [38, 39], respectively, for Be-like and B-like ions of astrophysical interest with the nuclear charge ranging up to $Z=54$. The calculated plasma rate coefficients for these ions are available from the Open ADAS database [40]. References to earlier theoretical work can be found in $[37,38]$.

Within the silicon isonuclear sequence of ions, experimental RR and DR results are available for Na-like $\mathrm{Si}^{3+}$ [4244], Be-like $\mathrm{Si}^{10+}$ [22], Li-like $\mathrm{Si}^{11+}[45,46]$ and bare $\mathrm{Si}^{14+}$ [47]. In addition, detailed measurements of recombination resonances have been conducted using X-ray spectroscopy of 
silicon ions in an electron-beam ion-trap (EBIT) [48]. The previous experimental data for $\mathrm{Si}^{10+}$ [22] cover a limited range of electron-ion collision energies of $0-43 \mathrm{eV}$. Thus, these data comprise only $\Delta N=0 \mathrm{DR}(2 s \rightarrow 2 p)$ and part of the $\Delta N=0$ TR $\left(2 s^{2} \rightarrow 2 p^{2}\right)$. In contrast, the present $\mathrm{Si}^{10+}$ measurements were carried out over a much wider collision energy range extending up to $2000 \mathrm{eV}$. This energy range covers all DR resonances including those associated with $\mathrm{K}$-shell excitation. Our resulting $\mathrm{Si}^{10+}$ plasma rate coefficient is accordingly valid for much higher temperatures including those where $\mathrm{Si}^{10+}$ forms in a collisionally ionized plasma. It should be noted, however, that our $\mathrm{Si}^{9+}$ DR plasma rate coefficient is only valid for lower temperatures including those where $\mathrm{Si}^{9+}$ forms in a photoionized plasma.

\section{Experiment}

The PR measurements were performed at the heavy-ion storage-ring, TSR [49], at the Max-Planck-Institut für Kernphysik (MPIK) in Heidelberg, Germany. The experimental procedures have been described before [50-53]. Therefore, we focus here on the details that are specific to the present measurements.

${ }^{29} \mathrm{Si}^{10+}$ and ${ }^{28} \mathrm{Si}^{9+}$ were provided by the MPIK tandem and linear accelerators and injected into TSR at energies of about 120 and $100 \mathrm{MeV}$, respectively. The choice of the less abundant isotope ${ }^{29} \mathrm{Si}$ with a natural abundance of only $4.7 \%$ for the measurements with $\mathrm{Si}^{10+}$ is motivated below (section 2.1). The stored ion currents were typically $10-20 \mu \mathrm{A}$ for $\mathrm{Si}^{10+}$ and $1 \mu \mathrm{A}$ for $\mathrm{Si}^{9+}$ after injection and from then on decreased exponentially as a function of storage time. The TSR electron cooler was used for phase-space cooling of the ion beam and as an electron target for the recombination measurements. The high-resolution electron target, which is installed at TSR in addition to the cooler, was not used for the present measurements since its photocathode provides much lower electron currents and consequently much lower recombination signal rates than the thermionic cathode of the cooler. The first dipole magnet behind the electron cooler was used to separate the recombined ions from the circulating beam. The recombined ions were detected by an appropriately placed single-particle detector [54] with nearly $100 \%$ efficiency.

During electron cooling the electron energy was set to the cooling energy $E_{\text {cool }}$ where electrons and ions move with the same velocity. This corresponds to zero collision energy in the electron-ion center-of-mass frame. The cooling energy was $2076 \mathrm{eV}$ for $\mathrm{Si}^{10+}$ and $1788 \mathrm{eV}$ for $\mathrm{Si}^{9+}$. After injection, the ions were continuously cooled for $2 \mathrm{~s}$ to reach optimal experimental conditions. For the recombination measurements the electron-ion collision energy $E$ was detuned from zero by changing the cooler cathode voltage. Each energy scan comprised typically $k_{\max }=300$ preselected electron-ion collision energies $E_{\text {meas }}^{(k)}$ with $k=1, \ldots, k_{\max }$. Between any two measurement steps the electron-ion collision energy was set to a reference value $E_{\text {ref }}$ and then to $E_{\text {cool }}$ to determine background and to maintain the ion beam quality, respectively. The intermediate cooling step was only applied at collision energies below $270 \mathrm{eV}$. It was left out at higher energies since the associated voltage jumps became too large for maintaining well controlled experimental conditions. There was a waiting time interval of $17 \mathrm{~ms}$ duration after each change of $E$ to allow the power supplies to settle to their new voltages. Then data were taken at $E_{\text {meas }}^{(k)}, E_{\text {ref }}$, and $E_{\text {cool }}$ for a dwell time interval of $25-50 \mathrm{~ms}$ duration. The recombination signal recorded at $E_{\text {ref }}$ was used for background determination. The entire injection-cooling-scanning sequence was repeated hundreds to thousands of times until the statistical uncertainties were reduced to the desired level. Multiple collision-energy scans with typically $50 \%$ overlap were used to cover the electron-ion collision energy ranges $0-2000 \mathrm{eV}$ for $\mathrm{Si}^{10+}$ and $0-50 \mathrm{eV}$ for $\mathrm{Si}^{9+}$.

Merged-beams rate coefficients (see e.g. [55]) were derived by normalizing the measured recombination count rate (after subtraction of background due to electron capture by the stored ions from residual gas particles) to the electron density in the cooler and to the stored-ion current and by taking the geometrical overlap of both beams into account [52]. In addition, a correction procedure was applied that accounts for the nonzero angles between the electron and the ion beam in the toroidal sections of the electron cooler [51]. The ion current was measured with a DC beam transformer with an accuracy of 1-2 $\mu \mathrm{A}$. Additionally, in order to measure also $\mathrm{Si}^{10+}$ currents below $1 \mu \mathrm{A}$, the count rate from the TSR beam profile monitor (BPM) was used as a proxy for the ion current. The BPM is based on residual gas ionization [56] and there is a linear relationship between BPM count rate and stored ion current. The constant of proportionality was calibrated repeatedly against the current transformer measurement at high stored ion currents. For $\mathrm{Si}^{9+}$, however, the ion currents were too low for a reliable calibration of the BPM signal. Therefore, the $\mathrm{Si}^{9+}$ rate coefficient scale was normalized to the absolute recombination rate coefficient at zero electron-ion collision energy. The latter was determined from a separate measurement of the storage lifetime of the ion beam following the procedures of [53], which were also employed in recent recombination experiments with $\mathrm{W}^{20+}$ [57] and $\mathrm{W}^{18+}$ [58] ions, where only very low ion currents were available. Here we tested the reliability of this method by performing a cross check with $\mathrm{Si}^{10+}$ ions. We found that the $\mathrm{Si}^{10+}$ recombination rate-coefficients from the beamlifetime and the ion-current measurements agreed with each other to within the experimental uncertainties. The uncertainty of the $\mathrm{Si}^{9+}$ merged-beams rate-coefficient scale is estimated to amount to $\pm 25 \%$ (at a $90 \%$ confidence level) [51]. In case of the Be-like $\mathrm{Si}^{10+}$ primary ion there is an additional $7 \%$ uncertainty due to an unknown fraction of metastable ions in the primary beam as discussed below. Adding the uncertainties in quadrature results in a $\pm 26 \%$ uncertainty of the $\mathrm{Si}^{10+}$ merged-beams rate-coefficient scale. The systematic uncertainty of the electron-ion collision energy scale is negligible at electron-ion collision energies below $\sim 1 \mathrm{eV}$ and increases with increasing energy. A 
conservative estimate [50] yields systematic uncertainties of $0.4,0.7$, and $1.5 \mathrm{eV}$ at electron-ion collision energies of 40 , 200 , and $1700 \mathrm{eV}$, respectively.

\subsection{Metastable primary ions}

Be-like ions are known to have long-lived $2 s 2 p{ }^{3} P_{J}$ levels $(J=0,1,2)$ which might have been present in the ion beam used for the measurements described here. The predicted lifetime of the ${ }^{28} \mathrm{Si}^{10+}(2 s 2 p){ }^{3} P_{0},{ }^{3} P_{1}$ and ${ }^{3} P_{2}$ levels are several 100 days [59], $2.747 \mu$ s and 0.4729 s [16] due to the dominant two-photon (E1M1), intercombination (E1), and magnetic quadrupole (M2) transitions, respectively. For nuclei with a nonzero magnetic moment, hyperfine quenching shortens the ${ }^{3} P_{0}$ lifetime by 6 orders of magnitude. Recent calculations yield a value of about $17 \mathrm{~s}$ for the hyperfine induced (HFI) lifetime of the ${ }^{29} \mathrm{Si}^{10+}\left(2 s 2 p{ }^{3} P_{0}\right)$ level $[17,60]$.

In fact, one motivation for the present recombination measurement was to derive the HFI ${ }^{3} P_{0}$ lifetime using the same approach as already successfully applied for the HFI lifetime of the $2 s 2 p{ }^{3} P_{0}$ level in Be-like ${ }^{47} \mathrm{Ti}^{18+}[25]$ and ${ }^{33} \mathrm{~S}^{12+}$ [23]. This approach requires the identification of DR resonances of ${ }^{3} P_{0}$ parent ions by comparing recombination spectra with different amounts of the $\mathrm{Si}^{10+}\left(2 s 2 p{ }^{3} P_{0}\right)$ parent ion. If such a resonance is present the visible resonance strength in the measured ${ }^{29} \mathrm{Si}^{10+}$ spectrum would be suppressed with increasing storage time due to the decaying ${ }^{3} P_{0}$ level. However, no significantly strong DR resonances associated with the initially metastable ${ }^{3} P_{0}$ level were found. This is attributed to the fact that DR resonances of the ${ }^{3} P_{0}$ parent ions have been found to be weak and to be blended with the rich DR resonance structure of the ${ }^{1} S_{0}$ ground state parent ions.

We estimated the ${ }^{3} P_{0}$ fraction in the parent beam by solving a set of rate equations [32] that accounts for the population and depopulation of the various ${ }^{29} \mathrm{Si}^{10+}$ levels by radiative transitions. The corresponding rates were taken from $[16,17]$ (table 1), and a Boltzmann distribution with a temperature of about $2100 \mathrm{eV}$ (corresponding to the ion energy at the last stripping foil in the accelerator) was chosen as the initial population of the levels. The resulting level populations are shown in figure 1. They turned out to be rather insensitive to the choice of initial populations. After an ion-storage time of $2 \mathrm{~s}$, only two levels remain significantly populated, i.e., the $2 s^{2}{ }^{1} S_{0}$ ground level and the $2 s 2 p{ }^{3} P_{0}$ first excited level with their fractional populations amounting to $93 \%$ and $7 \%$, respectively. At longer times, the $2 s 2 p{ }^{3} P_{0}$ level is depopulated by the $2 s 2 p{ }^{3} P_{0} \rightarrow 2 s^{2}{ }^{1} S_{0}$ HFI decay in ${ }^{29} \mathrm{Si}^{10+}$. Since data collection in the experiment started only after an initial cooling time of $2 \mathrm{~s}$, we assume in the following that the fraction of ions in the $2 s 2 p{ }^{3} P_{0}$ level was at most $7 \%$. This is a conservative estimate since the ${ }^{3} P_{0}$ fraction is predicted to have dropped to below $1 \%$ during the $\sim 30 \mathrm{~s}$ (see above) that was required to complete an energy scan.

The longest lived excited level of $\mathrm{Si}^{9+}$ is the $2 s^{2} 2 p{ }^{2} P_{3 / 2}$ level with a calculated lifetime of $0.324 \mathrm{~s}$ [41] (table 2). This lifetime is much shorter than the initial beam-cooling time of

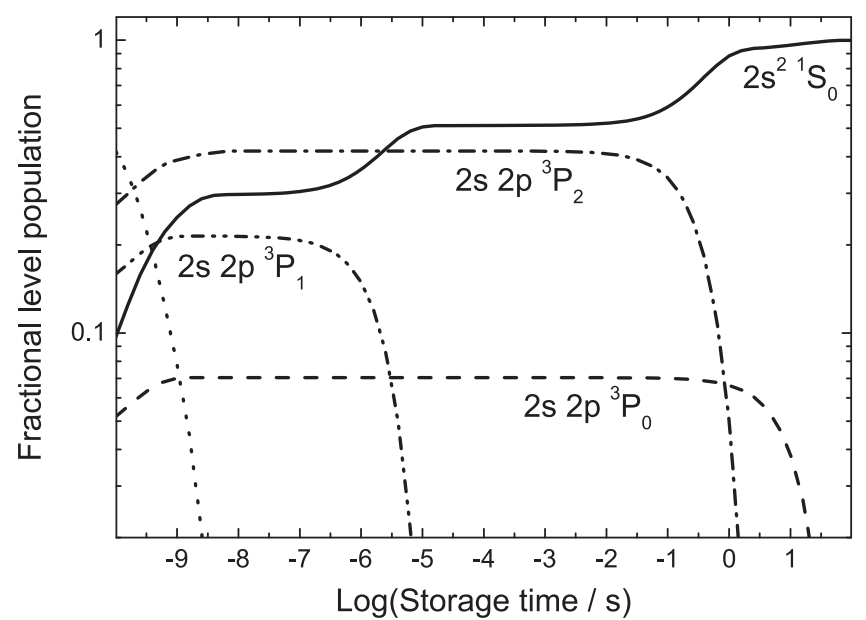

Figure 1. Calculated fractional ${ }^{29} \mathrm{Si}^{10+}$ level populations as a function of storage time using theoretical transition rates [16] (see also table 1) for the 116 lowest $\mathrm{Si}^{10+}$ levels. In addition, the rate for the hyperfine induced $2 s 2 p{ }^{3} P_{0} \rightarrow 2 s^{2}{ }^{1} S_{0}$ transition $\left(A_{\mathrm{HFI}}=0.06011 \mathrm{~s}^{-1}\right)$ was taken from [17]. The different lines correspond to the following levels: $2 s^{2}{ }^{1} S_{0}$ (-), $2 s 2 p^{3} P_{0}(---)$, $2 s 2 p{ }^{3} P_{1}(-\cdots-)$, and $2 s 2 p{ }^{3} P_{2}(-\cdots-)$. The dotted curve $(\cdots \cdots)$ is the sum of the fractional populations of the remaining 112 levels.

$2 \mathrm{~s}$. It is thus assumed that all $\mathrm{Si}^{9+}$ ions were in their ground level when data taking was started.

\section{Results}

\subsection{Merged-beams recombination rate coefficient for $\mathrm{Si}^{10+}$}

The measured merged-beams rate coefficients for the recombination of $\mathrm{Si}^{10+}$ ions are displayed in figures 2,3 , and 4 which comprise the energy ranges of DR associated with $2 s \rightarrow 2 p(\Delta N=0), 2 s \rightarrow 3 l, 4 l(\Delta N=1,2)$, and $1 s \rightarrow N^{\prime} l^{\prime}$ core excitations, respectively. The strongest resonances are associated with the $2 s^{2}{ }^{1} S_{0} \rightarrow 2 s 2 p{ }^{1} P_{1}$ core excitation with an excitation energy $E_{\text {exci }}=40.875 \mathrm{eV}$. In general, recombination resonance positions can be estimated from the Rydberg formula

$$
E_{\text {res }}(n)=E_{\text {exci }}-\mathcal{R} \frac{q^{2}}{n^{2}}
$$

with $\mathcal{R} \approx 13.6057 \mathrm{eV}$, the primary ion charge state $q=10$ and the excitation energies $E_{\text {exci }}$ from table 1 . The resulting calculated resonance positions are indicated by vertical bars in figures 2 and 3. Obviously the simple Rydberg formula works reasonably well for high- $n$ resonances where the interaction between the Rydberg electron and the excited core is only weak. The low- $n$ resonance structure, however, is dominated by fine-structure effects and the assignment of the measured structure becomes more complicated. More detailed insight is provided by comparing the measured data with results of theoretical calculations. To this end we have employed the Autostructure code [61]. In these calculations we have adjusted the DR series limits to observed excitation energies from the NIST Atomic Structure Data Base [15]. Our 


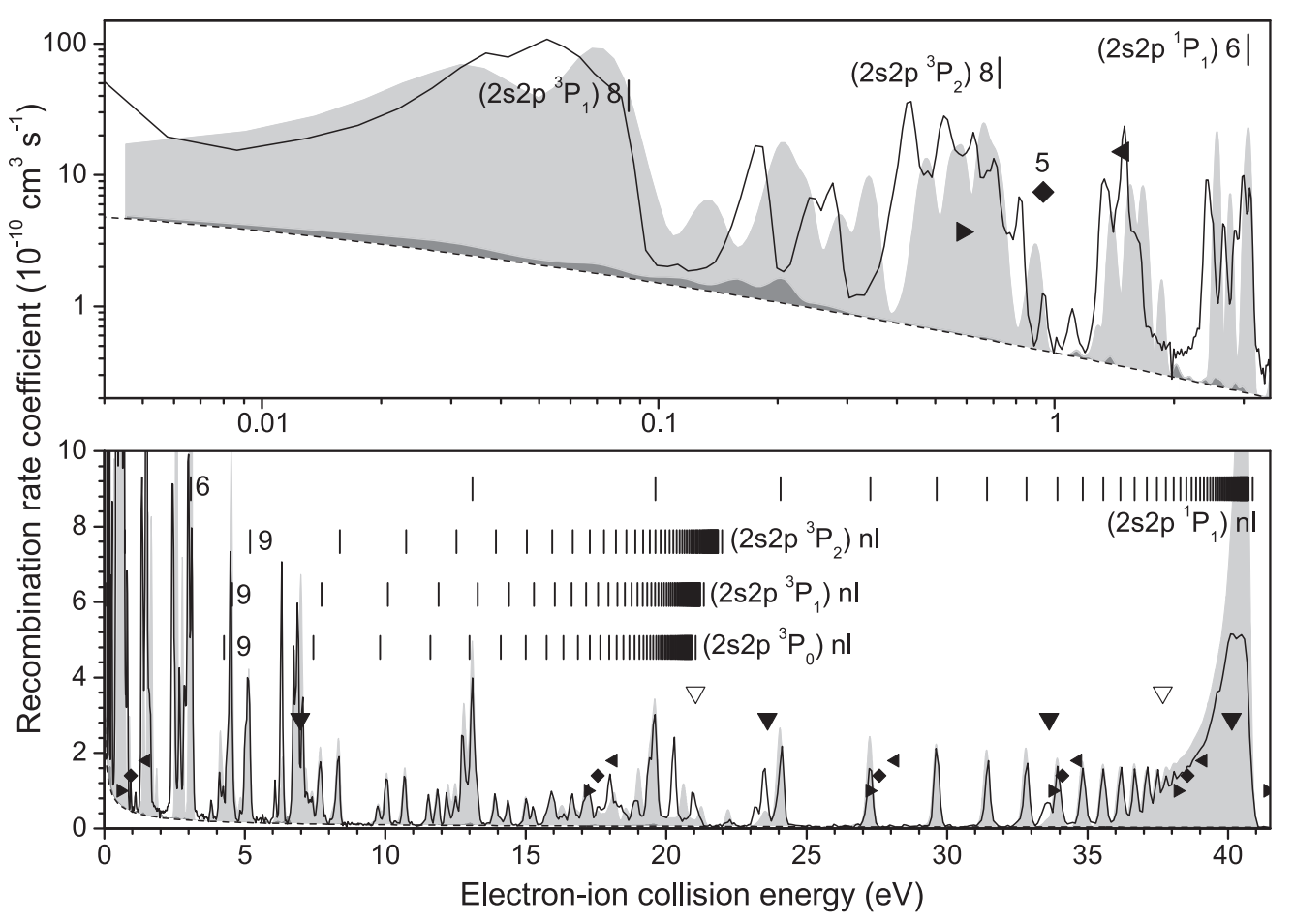

Figure 2. Measured merged-beams rate coefficient (solid black line) for PR of $\mathrm{Si}^{10+}$ in the energy range of the $2 \rightarrow 2 \Delta N=0 \mathrm{DR}$ resonances. The vertical bars denote DR resonance positions for ground-level ions calculated using equation (3) with $q=10$ and with the excitation energies taken from table 1 (NIST values). Calculated energies of TR resonances associated with $2 s^{2}+e^{-} \rightarrow 2 p^{2}{ }^{3} P_{0,1,2},{ }^{1} D_{2}$ and ${ }^{1} S_{0}$ excitations are marked by the following symbols: $\boldsymbol{\vee},, \mathbf{4}, \mathbf{\nabla}$ and $\nabla$, respectively. The calculated RR rate coefficient is shown as a dashed line. Results from DR and TR calculations with the AUTOSTRUCTURE code are shown as shaded areas. In these calculations an ion beam composition of 93\% $2 s^{2}{ }^{1} S_{0}$ ground level and 7\% $2 s 2 p{ }^{3} P_{0}$ metastable level was assumed. DR resonances associated with the excitation of the $2 s^{2}{ }^{1} S_{0}$ ground level (light shaded) and the $2 s 2 p{ }^{3} P_{0}$ metastable level are shown separately (dark shaded, only visible in the top panel).

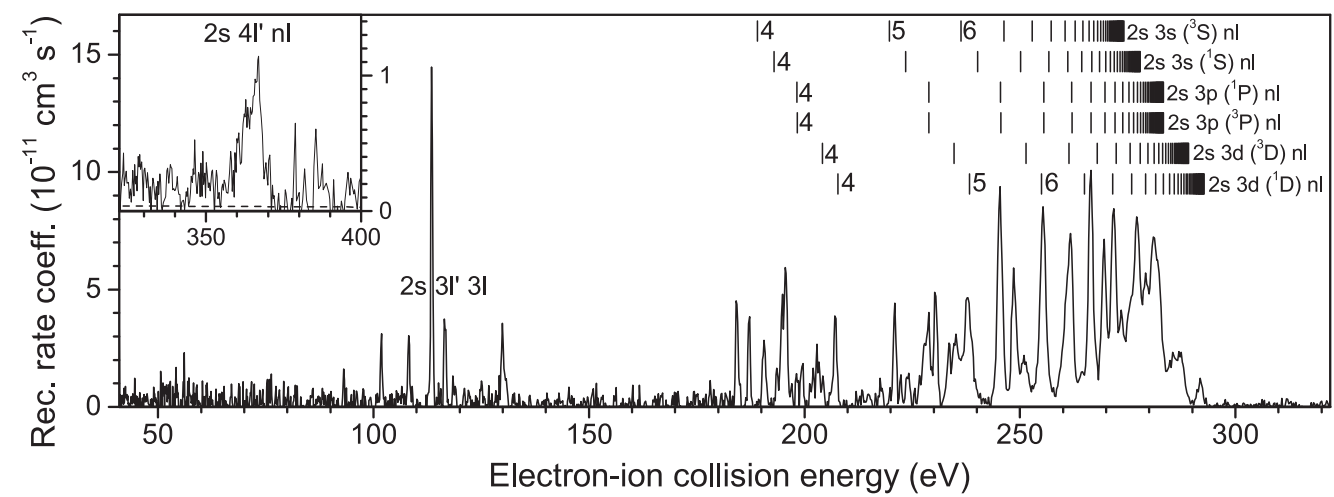

Figure 3. Measured merged-beams rate coefficient (solid black line) for $\mathrm{PR}$ of $\mathrm{Si}^{10+}$ in the energy range of DR resonances associated with $2 \rightarrow 3$ (main panel) and $2 \rightarrow 4$ (inset) core excitations. The vertical bars denote DR resonance positions of ground-level ions calculated using equation (3) with $q=10$ and the excitation energies from table 1 (NIST values). The dashed line in the inset is the calculated RR rate coefficient.

theoretical results for $\Delta N=0 \mathrm{DR}$ and TR are shown in figure 2 as shaded curves. For the comparison the theoretical cross sections were convoluted with the experimental electron velocity distribution which is characterized by the temperatures $T_{\|}$and $T_{\perp}$ parallel and perpendicular to the propagation direction of the electron beam [50]. For the comparison in figure $2, k_{\mathrm{B}} T_{\|}=93 \mu \mathrm{eV}$ and $k_{\mathrm{B}} T_{\perp}=11 \mathrm{meV}$ were used (with $k_{\mathrm{B}}$ being Boltzmann's constant) as determined by fits of theoretical line shapes to narrow DR resonances at electronion collision energies of 0.17 and $6.4 \mathrm{eV}$.

The calculated and measured merged-beams rate coefficients agree very well with one another over almost the entire energy range displayed in figure 2. At energies below $\sim 3 \mathrm{eV}$, there are small deviations in resonance positions and strengths. In addition, close to the $2 s 2 p\left({ }^{1} P_{1}\right) n l$ series limit, the calculated rate coefficient is much larger than the experimental 


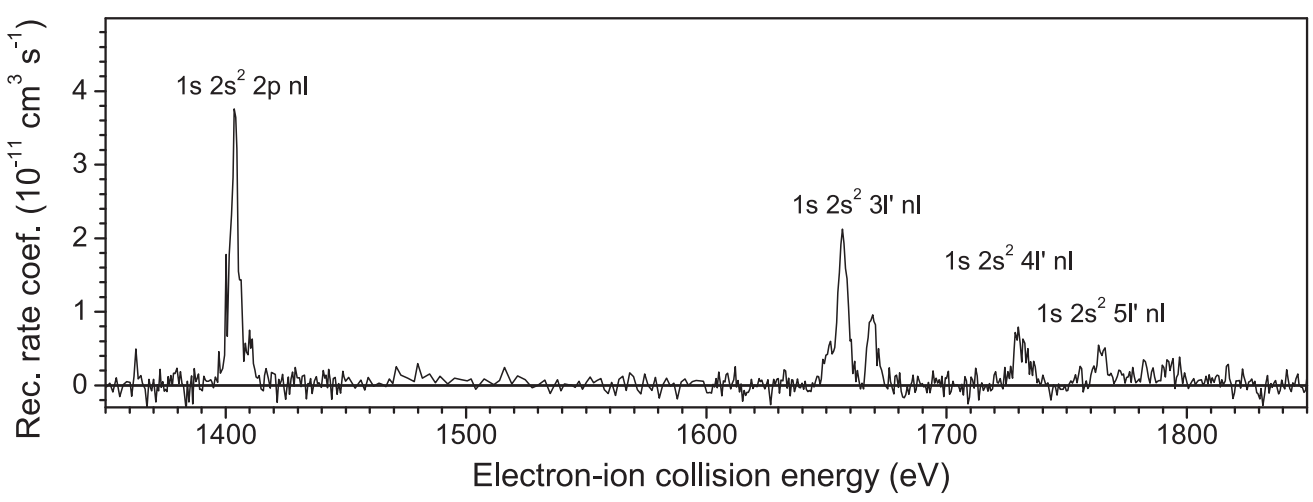

Figure 4. Measured merged-beams rate coefficient (solid black line) for $\mathrm{PR}$ of $\mathrm{Si}^{10+}$ in the energy range of DR resonances associated with K-shell excitations. Resonance groups are labelled by the according $1 s^{2} 2 s^{2} \rightarrow 1 s 2 s^{2} N^{\prime} l^{\prime}$ core excitations.

result. The origin of this latter discrepancy is well understood. It is due to field ionization of weakly bound high- $n$ Rydberg states which occurs primarily in the dipole bending magnets of the storage ring [52]. This field ionization effect has to be accounted for in the derivation of the plasma rate coefficient.

Apart from the dominating $2 s 2 p\left({ }^{1} P_{1}\right) n l$ Rydberg series, there are the weaker $2 s 2 p\left({ }^{3} P\right) n l$ series with their series limits at around $21.5 \mathrm{eV}$ (table 1) and a few isolated TR resonances such as the $2 p^{2}\left({ }^{1} S_{0}\right) 5 l$ resonance at $21.0 \mathrm{eV}$, the $2 p^{2}\left({ }^{1} D_{2}\right) 6 l$ resonance at $23.6 \mathrm{eV}$, and the $2 p^{2}\left({ }^{1} D_{2}\right) 7 l$ resonance at $33.6 \mathrm{eV}$. The role of TR in low-energy recombination of $\mathrm{Si}^{10+}$ has been studied already thoroughly by Orban et al [22]. They found that a large part of the resonance structure below $9 \mathrm{eV}$ and in the range $15-25 \mathrm{eV}$ can be attributed to TR and, thus, confirmed earlier findings on the importance of TR for Be-like ions [24].

The present AutostRUCTURE calculations were carried out for primary $\mathrm{Si}^{10+}$ ions in the $2 s^{2}{ }^{1} S_{0}$ ground level (light shaded area in figure 2) and in the long-lived $2 s 2 p{ }^{3} P_{0}$ metastable level (dark shaded area in figure 2) assuming a ratio of 93:7 for the two beam components (cf section 2.1). The calculations suggest that the contribution of the initial metastable ions to the measured $\Delta N=0 \mathrm{DR}+\mathrm{TR}$ rate coefficient is practically negligible. Nevertheless, in the derivation of a pure ground-level plasma rate coefficient the (assumed) $7 \%$ metastable fraction introduces a corresponding uncertainty in the normalization to the ion current which has been taken into account in our error budget as described above.

In contrast to the $\Delta N=0$ resonances discussed so far, the DR resonance structure associated with $2 s \rightarrow 3 l \Delta N=1$ core excitations (figure 3 ) is less regular. This is due to the many mutually overlapping Rydberg series of DR resonances. The $\Delta N=1$ series limits are between 274 and $293 \mathrm{eV}$ (table 1). At higher energies of about $370 \mathrm{eV}$, resonances associated with $2 s \rightarrow 4 l \Delta N=2$ core excitations are visible. They are an order of magnitude smaller than the $\Delta N=1$ resonances. Contributions associated with even higher excitations were not observed. They can be expected to be negligibly small. The cumulative strength of the high- $n \Delta N=1$ resonances does not pile up as much as for the $\Delta N=0$ Rydberg series because the $\Delta N=1$ resonance strength decreases much faster with increasing $n$ than the $\Delta N=0$ resonance strength. Therefore, $\Delta N=1 \mathrm{DR}$ is much less affected by field ionization than $\Delta N=0 \mathrm{DR}$.

The highest electron-ion collision energies studied were in the range 1300-2000 eV where DR resonances occur that are associated with the excitation of a $1 \mathrm{~s}$ electron to a higher principal shell $N^{\prime}$ (figure 4). The $1 s 2 s^{2} N^{\prime} l^{\prime} n l$ DR resonance strengths decrease rapidly with increasing $N^{\prime}$ and increasing $n$. There is no significant DR resonance strength beyond an electron-ion collision energy of $\sim 1800 \mathrm{eV}$ since DR cannot occur at energies beyond the threshold for direct K-shell ionization of the $\mathrm{Si}^{10+}$ parent ion.

\subsection{Merged-beams recombination rate coefficient for $\mathrm{Si}^{9+}$}

Experimental and theoretical data for RR and $\Delta N=0 \mathrm{DR}$ of boronlike $\mathrm{Si}^{9+}$ ions are presented in figure 5 . The theoretical cross sections were calculated using the AutOSTRUCTURE code and merged-beams rate coefficients were subsequently derived by convolution with the experimental electron velocity distribution using $k_{\mathrm{B}} T_{\|}=180 \mu \mathrm{eV}$ and $k_{\mathrm{B}} T_{\perp}=12 \mathrm{meV}$. The $\mathrm{Si}^{9+}$ DR spectrum exhibits three strong Rydberg series associated with the formation of doublet terms of $2 s 2 p^{2}$ core excited levels (table 2). At energies below $1 \mathrm{eV}$ DR Rydberg resonances can be formed via $2 s^{2} 2 p^{2} P_{1 / 2} \rightarrow 2 s^{2} 2 p{ }^{2} P_{3 / 2}$ fine-structure core excitations [62] with the corresponding series limit occurring at $0.867 \mathrm{eV}$. As for $\mathrm{Si}^{10+}$ the theoretical calculations for $\mathrm{Si}^{9+}$ agree also well the experimental data, except for the resonance structure below $\sim 2 \mathrm{eV}$ which is notoriously difficult to calculate accurately because the multiply excited levels just above the autoionization threshold of the recombined ion are subject to strong correlation effects. These are apparently stronger for $\mathrm{Si}^{9+}$ (figure 5) than for $\mathrm{Si}^{10+}$ (figure 2) because of the presence of an additional electron. The differences close to the Rydberg series limits are due to field ionization effects as already discussed above for $\mathrm{Si}^{10+}$.

\subsection{Plasma rate coefficients}

For the derivation of plasma rate coefficients of $\mathrm{Si}^{9+}$ and $\mathrm{Si}^{10+}$ ions from the measured merged-beams recombination rate coefficients we follow the procedure described by 


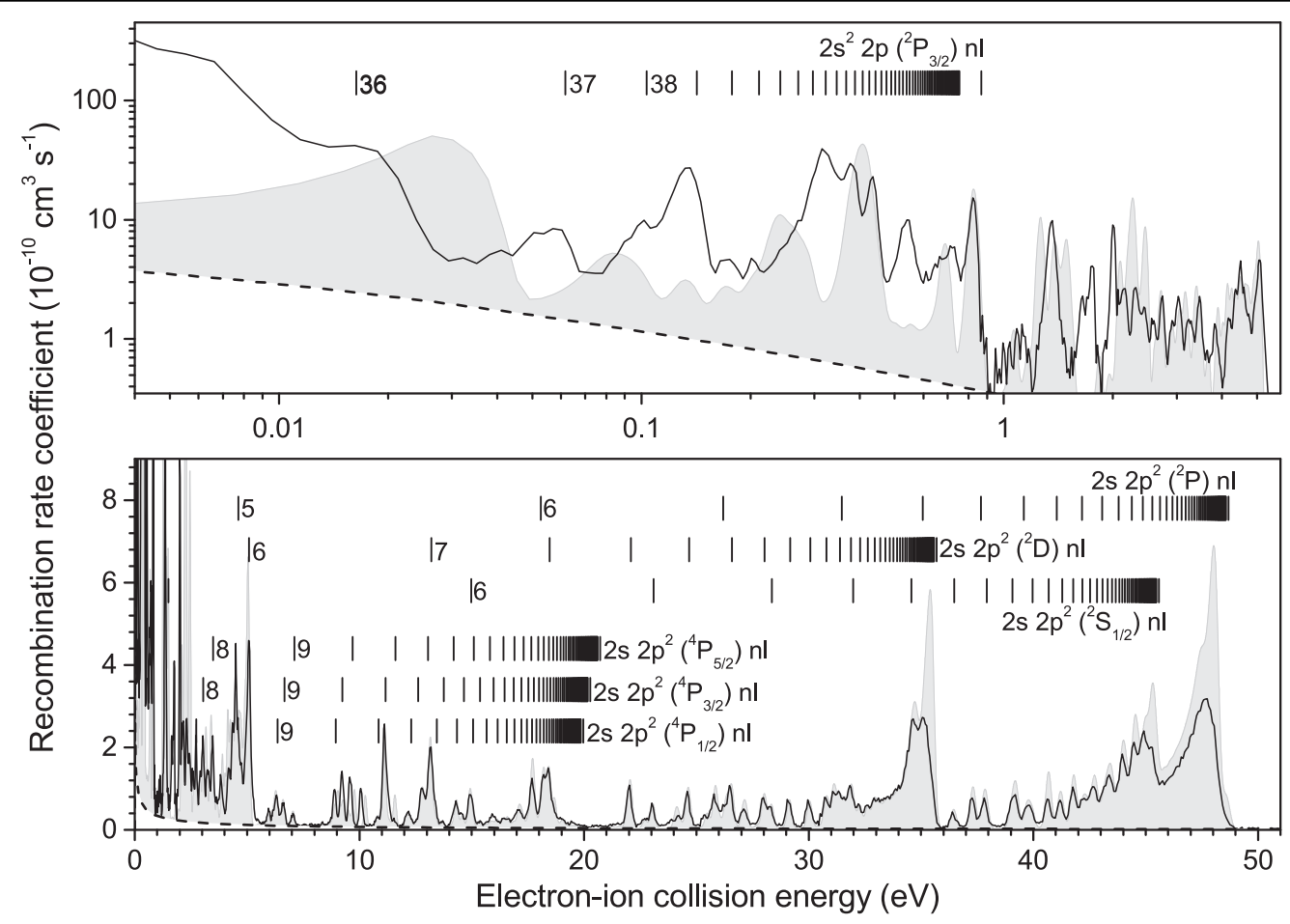

Figure 5. Measured merged-beams rate coefficient (solid black line) for PR of $\mathrm{Si}^{9+}$ in the energy range of DR resonances associated with intra L-shell $\Delta N=0$ core excitations. The black vertical bars denote DR resonance positions calculated using equation (3) with $q=9$ and with the excitation energies taken from table 2 (NIST values). Results from DR calculations with the AutosTRUCTURE code are shown as a shaded curve on top of the small calculated RR-rate coefficient (dashed line).

Schippers et al [52]. Accordingly, field ionization is accounted for by replacing the measured rate-coefficients by appropriately scaled AuTOSTRUCTURE results in the energy ranges close to the $\Delta N=0$ Rydberg series limits. No such corrections are required for the $\Delta N>0$ series limits, which are much less affected by field ionization as explained above. Plasma rate coefficients for $\mathrm{DR}(+\mathrm{TR})$ of ground-level ions were then derived by first subtracting the calculated $R R$ rate coefficients from the measured data and by then convoluting the remaining DR(+TR) merged-beams rate coefficients by an isotropic Maxwellian. The resulting $\mathrm{Si}^{10+}$ and $\mathrm{Si}^{9+} \mathrm{DR}$ rate coefficients in a plasma are plotted in figures 6 and 7 as functions of plasma temperature. Their systematic uncertainties of $26 \%$ and $25 \%$, respectively, at a $90 \%$ confidence level, correspond directly to the systematic uncertainties of the underlying experimental merged-beams rate coefficients.

The present experimentally derived $\mathrm{Si}^{10+}$ plasma $\mathrm{DR}+$ TR rate coefficient agrees with the most recent theoretical result [37] within the experimental uncertainties over almost the entire plotted (figure 6) temperature range of 0.5-5000 $\mathrm{eV}$. In particular, there is good agreement in the temperature ranges where $\mathrm{Si}^{10+}$ forms in photoionized plasmas (PP) and collisionally ionized plasmas $(\mathrm{CP})$. These temperature ranges are shaded in figure 6 and have been estimated on the basis of charge balance calculations by Kallman and Bautista [63] and by Bryans et al [8], respectively. Also shown in figure 6 are the individual contributions by the processes listed in equation (1). Clearly, $\Delta N>0$ DR contributes significantly to

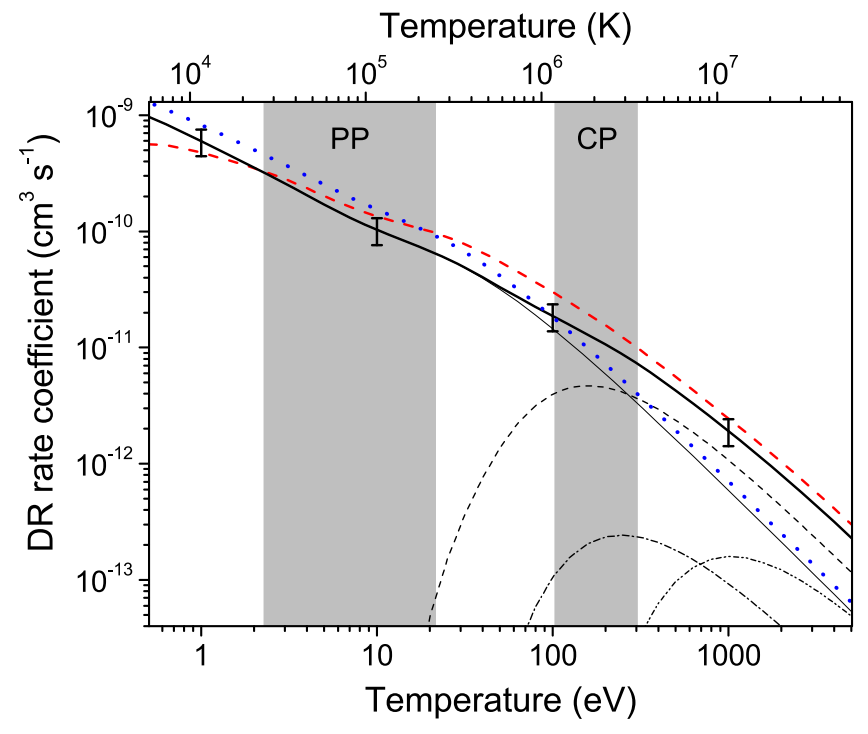

Figure 6. Rate coefficients for DR of $\mathrm{Si}^{10+}\left(2 s^{2}{ }^{1} S_{0}\right)$ ions in a plasma. The thick full line is our present experimentally derived rate coefficient. The error bars denote the $\pm 26 \%$ systematic uncertainty at a $90 \%$ confidence level. DR contributions associated with $2 \rightarrow 2,2 \rightarrow 3,2 \rightarrow 4$ and $1 \rightarrow N^{\prime}$ excitations are given as straight, dashed, dash-dotted and dot-dash-dotted thin lines. The (blue) dotted line is the result for $\Delta N=0$ DR by Orban et al [22]. The most recent theoretical result [37] is shown as (red) thick dashed line. Approximate temperature ranges where $\mathrm{Si}^{10+}$ is expected to form in photoionized plasmas (PP) [63] and collisionally ionized plasmas (CP) [8] are indicated as gray shaded areas. 


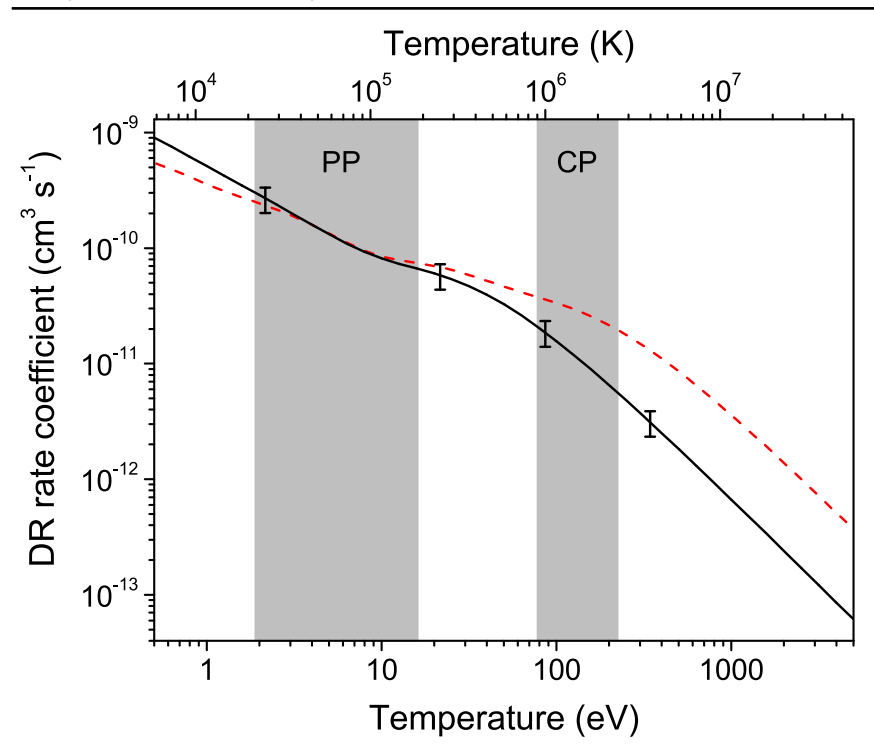

Figure 7. Rate coefficients for DR of $\mathrm{Si}^{9+}\left(2 s^{2} 2 p^{2} P_{1 / 2}\right)$ ions in a plasma. The thick full line is our present experimentally derived $\Delta N=0$ DR rate coefficient. The error bars denote the $\pm 25 \%$ uncertainty at a $90 \%$ confidence level. The most recent theoretical results [38] comprising $\Delta N=0$ and $\Delta N>0$-DR is shown as a dashed line. Approximate temperature ranges where $\mathrm{Si}^{9+}$ is expected to form in photoionized plasmas (PP) [63] and collisionally ionized plasmas (CP) [8] are indicated as gray shaded areas.

the total DR rate coefficient in the $\mathrm{CP}$ temperature range. The previous experimentally derived result of Orban et al [22] for $\Delta N=0 \mathrm{DR}+\mathrm{TR}$ (dotted line in figure 6) agrees with the present $\Delta N=0 \mathrm{DR}+\mathrm{TR}$ rate coefficient within the combined experimental uncertainties.

Within the $25 \%$ experimental uncertainty (at a $90 \%$ confidence level), the $\mathrm{Si}^{9+}$ DR rate coefficient agrees with the most recent theoretical result [38] only for temperatures between 1.5 and $30 \mathrm{eV}$ comprising the entire PP temperature range (figure 7). At higher temperatures the experimental result is significantly lower than the theoretical prediction. This is due to the neglect of $\Delta N>0$ DR in the experimentally derived rate coefficient of $\mathrm{Si}^{9+}$. Because of time constraints there were no measurements in the corresponding electron-ion collision energy ranges.

For convenient use of our results in plasma modeling codes, we provide a simple parametrization where we have fitted the function

$$
\alpha_{\mathrm{DR}}(T)=T^{-3 / 2} \sum_{i} c_{i} \exp \left(-E_{i} / T\right)
$$

to our experimentally derived $\mathrm{DR}(+\mathrm{TR})$ plasma rate coefficients. The fit parameters $c_{i}$ and $E_{i}$ are listed in table 3 . For plasma temperatures $T$ between $10^{2}$ and $5 \times 10^{7} \mathrm{~K}$, the fits deviate by less than $2 \%$ from the experimentally derived curves.

\section{Summary and conclusions}

Rate coefficients for dielectronic recombination of Be-like $\mathrm{Si}^{10+}$ and $\mathrm{B}$-like $\mathrm{Si}^{9+}$ have been measured at a heavy ion storage ring. For $\mathrm{Si}^{10+}$ the energy range has been greatly extended compared to the previous measurements by Orban
Table 3. Parameters for the parametrization (equation (4)) of the experimentally derived rate coefficients for $\mathrm{DR}+\mathrm{TR}$ of $\mathrm{Si}^{10+}\left(2 s^{2}{ }^{1} S_{0}\right)$ and for $\Delta N=0 \mathrm{DR}$ of $\mathrm{Si}^{9+}\left(2 s^{2} 2 p^{2} P_{1 / 2}\right)$ in a plasma. Numbers in square brackets denote powers of 10 . The parameters are valid for plasma temperatures between $10^{2}$ and $5 \times 10^{7} \mathrm{~K}$.

\begin{tabular}{lccccc}
\hline & \multicolumn{2}{c}{$\mathrm{Si}^{10+}$} & & \multicolumn{2}{c}{$\mathrm{Si}^{9+}$} \\
\cline { 2 - 3 } \cline { 5 - 6 } & $c_{i}\left(\mathrm{~cm}^{3} \mathrm{~s}^{-1} \mathrm{~K}^{3 / 2}\right)$ & $E_{i}(\mathrm{~K})$ & & $c_{i}\left(\mathrm{~cm}^{3} \mathrm{~s}^{-1} \mathrm{~K}^{3 / 2}\right)$ & $E_{i}(\mathrm{~K})$ \\
\hline 1 & $1.932[-5]$ & $2.996[1]$ & & $4.438[-5]$ & $3.837[1]$ \\
2 & $4.999[-6]$ & $5.778[1]$ & & $4.577[-5]$ & $9.834[1]$ \\
3 & $1.132[-4]$ & $5.823[2]$ & & $5.189[-5]$ & $9.261[2]$ \\
4 & $9.121[-5]$ & $1.477[3]$ & & $5.511[-4]$ & $4.656[3]$ \\
5 & $7.094[-4]$ & $6.760[3]$ & & $1.183[-3]$ & $2.507[4]$ \\
6 & $1.576[-3]$ & $2.818[4]$ & & $3.148[-3]$ & $1.161[5]$ \\
7 & $3.738[-3]$ & $1.106[5]$ & & $2.219[-2]$ & $4.584[5]$ \\
8 & $1.801[-2]$ & $4.200[5]$ & & & \\
9 & $5.727[-2]$ & $2.864[6]$ & & \\
10 & $3.160[-2]$ & $1.701[7]$ & & \\
\hline
\end{tabular}

et al [22]. The experimental energy range covered by the present experiment with $\mathrm{Si}^{10+}$ comprises the highest-energy DR resonances associated with K-shell core excitation. Correspondingly, the experimentally derived plasma rate coefficient for $\mathrm{DR}+\mathrm{TR}$ of $\mathrm{Si}^{10+}$ is valid for all temperatures. Within the experimental uncertainties it agrees with the most recent theoretical result [37]. The present result for $\Delta N=0$ $\mathrm{DR}+\mathrm{TR}$ also agrees with the previous experimentally derived rate coefficient [22] which was measured at another heavy-ion storage ring. This agreement (within the combined experimental uncertainties) demonstrates the reliability of the storage-ring technique. One of the hallmarks of this technique is that it allows for the preparation of ions in well defined energy levels. This has been exploited in particular for reducing the $\mathrm{Si}^{10+}$ ion beam contamination by long-lived $2 s 2 p{ }^{3} P$ metastable levels to almost insignificance.

For $\mathrm{Si}^{9+}$ only low-energy $\Delta N=0 \mathrm{DR}$ was measured. The experimentally derived plasma rate coefficient agrees with the most recent theoretical result [38] in the temperature range where $\mathrm{Si}^{9+}$ forms in a photoionized plasma. Our result may thus be applied in charge balance calculations for, e.g., active galactic nuclei [64].

Our experimental results benchmark state-of-the-art theoretical calculations for few-electron ions. The good agreement found provides confidence in the theoretical methods typically used for generation of $\mathrm{DR}(\mathrm{TR})$ plasma rate coefficients. It should be kept in mind however, that the accuracy of these theoretical methods is much more limited for many-electron ions as is shown by recent investigations of electron-ion recombination of complex open- $4 f$-shell ions [55, 57, 58, 65, 66].

\section{Acknowledgments}

We would like to thank the MPIK accelerator and TSR crews for their excellent support during the experiments. Financial support by the Max-Planck-Gesellschaft and by the Deutsche Forschungsgemeinschaft (DFG, contract no. Schi 378/8-1) is 
gratefully acknowledged. MH, ON, and DWS were supported in part by the NASA Solar Heliospheric Physics program.

\section{References}

[1] Asplund M, Grevesse N, Sauval A J and Scott P 2009 Annu. Rev. Astron. Astrophys. 47481

[2] Aguirre A, Schaye J, Kim T S, Theuns T, Rauch M and Sargent W L W 2004 Astrophys. J. 60238

[3] Doschek G A and Feldman U 2010 J. Phys. B 43232001

[4] Güdel M and Nazé Y 2009 Astron. Astrophys. Rev. 17309

[5] Decourchelle A, Sauvageot J L, Audard M, Aschenbach B, Sembay S, Rothenflug R, Ballet J, Stadlbauer T and West R G 2001 Astron. Astrophys. 365 L218

[6] Sako M, Kahn S M, Paerels F and Liedahl D A 2000 Astrophys. J. Lett. 543 L115

[7] Simcoe R A, Sullivan P W, Cooksey K L, Kao M M, Matejek M S and Burgasser A J 2012 Nature 49279

[8] Bryans P, Badnell N R, Gorczyca T W, Laming J M, Mitthumsiri W and Savin D W 2006 Astrophys. J. Suppl. Ser. 167343

[9] Kallman T 2010 Space Sci. Rev. 157177

[10] Ballance C P, Loch S D, Foster A R, Smith R K, Witthoeft M C and Kallman T R 2013 Fus. Sci. Technol. 63358

[11] Savin D W 2007 J. Phys.: Conf. Ser. 88012071

[12] Schippers S 2009 J. Phys.: Conf. Ser. 163012001

[13] Schippers S, Lestinsky M, Müller A, Savin D W, Schmidt E W and Wolf A 2010 Int. Rev. At. Mol. Phys. 1109

[14] Hahn M 2014 J. Phys.: Conf. Ser. 488012050

[15] Kramida A, Ralchenko Y, Reader J and NIST ASD Team 2014 NIST Atomic Spectra Database (version 5.2) (http:// physics.nist.gov/asd)

[16] Wang K et al 2015 Astrophys. J. Suppl. Ser. 21816

[17] Cheng K T, Chen M H and Johnson W R 2008 Phys. Rev. A 77 052504

[18] Fogle M, Badnell N R, Glans P, Loch S D, Madzunkov S, Abdel-Naby S A, Pindzola M S and Schuch R 2005 Astron. Astrophys. 442757

[19] Ali S, Orban I, Mahmood S, Loch S D and Schuch R 2013 Astron. Astrophys. 557 A2

[20] Orban I, Böhm S, Loch S and Schuch R 2008 Astron. Astrophys. 489829

[21] Schippers S, Schnell M, Brandau C, Kieslich S, Müller A and Wolf A 2004 Astron. Astrophys. 4211185

[22] Orban I, Loch S D, Böhm S and Schuch R 2010 Astrophys. J. 7211603

[23] Schippers S et al 2012 Phys. Rev. A 85012513

[24] Schnell M et al 2003 Phys. Rev. Lett. 91043001

[25] Schippers S, Schmidt E W, Bernhardt D, Yu D, Müller A, Lestinsky M, Orlov D A, Grieser M, Repnow R and Wolf A 2007 Phys. Rev. Lett. 98033001

[26] Schippers S, Schmidt E W, Bernhardt D, Yu D, Müller A, Lestinsky M, Orlov D A, Grieser M, Repnow R and Wolf A 2007 J. Phys.: Conf. Ser. 58137

[27] Savin D W et al 2006 Astrophys. J. 6421275

[28] Orlov D A, Krantz C, Bernhardt D, Brandau C, Hoffmann J, Müller A, Ricsóka T, Schippers S, Shornikov A and Wolf A 2009 J. Phys.: Conf. Ser. 163012058

[29] Bernhardt D et al 2015 J. Phys. B 48144008

[30] Ali S, Orban I, Mahmood S, Altun Z, Glans P and Schuch R 2012 Astrophys. J. 753132

[31] Mahmood S, Orban I, Ali S, Glans P, Bleda E A, Altun Z and Schuch R 2013 Astrophys. J. 77178

[32] Lestinsky M et al 2012 Astrophys. J. 75840

[33] Gao H, DeWitt D R, Schuch R, Zong W, Asp S and Pajek M 1995 Phys. Rev. Lett. 754381
[34] DeWitt D R, Schuch R, Gao H, Zong W, Asp S, Biedermann C, Chen M H and Badnell N R 1996 Phys. Rev. A 532327

[35] Savin D W et al 2003 Astrophys. J. Suppl. Ser. 147421

[36] Krantz C, Orlov D A, Bernhardt D, Brandau C, Hoffmann J, Müller A, Ricsóka T, Ricz S, Schippers S and Wolf A 2009 J. Phys.: Conf. Ser. 163012059

[37] Colgan J, Pindzola M S, Whiteford A D and Badnell N R 2003 Astron. Astrophys. 412597 (http://amdpp.phys.strath.ac. uk/tamoc/DATA/)

[38] Altun Z, Yumak A, Badnell N R, Colgan J and Pindzola M S 2004 Astron. Astrophys. 420775 (http://amdpp.phys.strath. ac.uk/tamoc/DATA/)

[39] Altun Z, Yumak A, Badnell N R, Colgan J and Pindzola M S 2005 Astron. Astrophys. 433395

[40] Open ADAS 2015 Atomic Data and Analysis Structure http:// open.adas.ac.uk

[41] Rynkun P, Jönsson P, Gaigalas G and Fischer C F 2012 At. Data Nucl. Data Tables 98481

[42] Orban I, Glans P, Altun Z, Lindroth E, Källberg A and Schuch R 2006 Astron. Astrophys. 459291

[43] Orban I, Lindroth E, Glans P and Schuch R 2007 J. Phys. B 401063

[44] Schmidt E W et al 2007 Phys. Rev. A 76032717

[45] Kenntner J et al 1995 Nucl. Instrum. Methods B 98142

[46] Bartsch T et al 1997 Phys. Rev. Lett. 792233

[47] Gao H, Schuch R, Zong W, Justiniano E, DeWitt D R, Lebius H and Spies W 1997 J. Phys. B 30 L499

[48] Baumann T M, Harman Z, Stark J, Beilmann C, Liang G, Mokler P H, Ullrich J and Crespo López-Urrutia J R 2014 Phys. Rev. A 90052704

[49] Grieser M et al 2012 Eur. Phys. J. ST 2071

[50] Kilgus G, Habs D, Schwalm D, Wolf A, Badnell N R and Müller A 1992 Phys. Rev. A 465730

[51] Lampert A, Wolf A, Habs D, Kenntner J, Kilgus G, Schwalm D, Pindzola M S and Badnell N R 1996 Phys. Rev. A 531413

[52] Schippers S, Müller A, Gwinner G, Linkemann J, Saghiri A A and Wolf A 2001 Astrophys. J. 5551027

[53] Novotný O et al 2012 Astrophys. J. 75357

[54] Rinn K, Müller A, Eichenauer H and Salzborn E 1982 Rev. Sci. Instrum. $\mathbf{5 3} 829$

[55] Müller A 2015 Atoms 3120

[56] Hochadel B, Albrecht F, Grieser M, Habs D, Schwalm D, Szmola E and Wolf A 1994 Nucl. Instrum. Methods A 343401

[57] Schippers S et al 2011 Phys. Rev. A 83012711

[58] Spruck K et al 2014 Phys. Rev. A 90032715

[59] Fritzsche S, Surzhykov A and Volotka A 2015 New. J. Phys. 17103009

[60] Andersson M, Zou Y, Hutton R and Brage T 2009 Phys. Rev. A 79032501

[61] Badnell N 2011 Comput. Phys. Commun. 1821528 (http:// amdpp.phys.strath.ac.uk/autos/)

[62] Savin D W, Bartsch T, Chen M H, Kahn S M, Liedahl D A, Linkemann J, Müller A, Schippers S, Schmitt M, Schwalm D and Wolf A 1997 Astrophys. J. 489 L115

[63] Kallman T and Bautista M 2001 Astrophys. J. Suppl. Ser. 133221

[64] Müller A and Schippers S 2012 Unravelling the mysteries of matter surrounding supermassive black holes Atomic Processes in Basic and Applied Physics (Springer Series on Atomic, Optical, and Plasma Physics vol 68) ed V Shevelko and H Tawara (Berlin, Heidelberg: Springer) ch 2

[65] Badnell N R, Ballance C P, Griffin D C and O'Mullane M 2012 Phys. Rev. A 85052716

[66] Dzuba V A, Flambaum V V, Gribakin G F, Harabati C and Kozlov M G 2013 Phys. Rev. A 88062713 